Recebido em 10/2019. Aceito para publicação em 11/2020.

\title{
AVALIAÇÃO DA PRÁTICA MINDFULNESS EM CIDADES DO VALE DO PARAÍBA
}

\section{EVALUATION OF MINDFULNESS PRACTICE IN CITIES OF VALE DO PARAÍBA}

\author{
Mariana de Oliveira Corrêa ${ }^{1}$ \\ Joyce Nascimento Santos ${ }^{2}$ \\ Josne Carla Paterno ${ }^{3}$
}

Resumo: A técnica de Meditação de Atenção Plena (Mindfulness) é definida como um método de atenção na experiência do momento presente de forma receptiva sugerindo uma nova postura por meio de um entendimento consciente do que acontece à sua volta, deste modo, diminuindo os fatores emocionais e fisiológicos, intervindo por meio da reatividade afetiva com benefícios mentais e físicos na saúde. Para isto, foi realizado uma análise quantitativa do controle de estresse com a prática Mindfulness entre duas cidades do Vale do Paraíba visto a escassez de evidencias iniciais na região, o estudo inicial foi realizado em 8 sessões em uma amostra delimitada aleatoriamente de 20 participantes (8 homens e 12 mulheres). Os resultados demonstraram o potencial que a prática possibilitou nas duas amostras avaliadas, com a melhora nos indicadores de estresse após o período da prática $(P=0,022$ e $P=0,003)$, além disso, foi possivel avaliar o potencial de controle que a prática proporcionou $(P=0,002)$. Portanto, os resultados obtidos indicam o potencial de melhora nos indicadores analisados e que a prática contínua do método de Atenção PlenaMindfulness, influencia na qualidade de vida dos indivíduos da sociedade e propicia bem-estar.

Palavras-chave: Meditação; Mindfulness; Medicina Tradicional Chinesa.

\begin{abstract}
The Mindfulness Meditation technique is defined as a method of attention in the experience of the present moment in a receptive way, suggesting a new posture by a conscious understanding of what happens around you, thus decreasing emotional and physiological factors, intervening by affective reactivity with mental and physical health benefits. For this, a quantitative analysis of stress control with the Mindfulness practice was conducted in two cities in Vale do Paraíba, given the scarcity of initial evidence in the region, and the initial study was conducted in 8 sessions in a randomly delimited sample of 20 participants ( 8 men and 12 women). The results demonstrated the potential that the practice made possible in the two samples evaluated, with the improvement in stress indicators after the period of practice $(P=$ 0.022 and $P=0.003$ ); in addition, it was possible to evaluate the potential of control that the practice provided $(P=0.002)$. Therefore, the results obtained indicate the potential for improvement in the analyzed indicators, and that the continuous practice of the Mindfulness Full Attention method influences the quality of life of individuals in society and promotes well-being.
\end{abstract}

Keywords: Meditation; Mindfulness; Traditional Chinese medicine.

\section{INTRODUÇÂO}

A medicina comportamental integrou a técnica de Atenção Plena (Mindfulness), a partir de estudos feitos por Kabat-Zinn (1982), considerado o pai do Mindfulness (LOPES; CASTRO; NEUFELD, 2012). A técnica simples, caracteriza-se por fechar os

\footnotetext{
${ }^{1}$ Faculdade Ciências da Saúde-FCS, Universidade do Vale do Paraíba - Univap, Brasil. E-mail: marianaoliveirac98@gmail.com.

2 Faculdade Ciências da Saúde-FCS, Universidade do Vale do Paraíba - Univap, Brasil. Especialização em Educação pela Universidade do Estado da Bahia - UNEB, Brasil. E-mail: santosnjoyce@gmail.com.

${ }^{3}$ Docente da Faculdade Ciências da Saúde-FCS, Universidade do Vale do Paraíba - Univap, Brasil. Especialização em Gestão, pela Faculdade Zacarias de Goés - FAZAG, Brasil. E-mail: josnepaterno@gmail.com.
} 
olhos e realizar respirações conscientes, não sendo necessário a realização de nenhuma atividade especial além de observar as sensações de seu corpo e mente no momento presente, sem julgamentos (CRESWELL, 2017; KABAT-ZINN, 1990). A origem do termo utilizado, deriva do Oriente, nas linhagens budistas de meditação, que proporcionou ao mestre Kabat a fundação do programa mais tradicional e estudado do mundo, Mindfulness Based Stress Reduction - MBSR, no Centro Médico da Universidade de Massachusetts (KABAT-ZINN, 1990).

Devido à mudança de padrões ao longo das décadas, os indivíduos adquiriram uma rotina estressante (KABAT-ZINN, 1990). Afinal, durante o cotidiano nossa mente prevê infelicidades subsequentes, que involuntariamente ocasiona uma parcela da sociedade a se condenar pela presença de pensamentos e sentimentos negativos, ao longo do tempo, essas emoções vão sendo acumuladas e sobrecarregam o indivíduo (LINDSAY; CRESWALL, 2017; HAYES et al., 2004; KABAT-ZINN, 1990). É do comportamento humano, a prática de se esquivar das situações e sentimentos dolorosos, na ilusão da presença de plenitude em todos os aspectos de suas vidas, ao invés de lidar com as razões de sofrimento (HAYES et al., 2004; ROEMER; BORKOVEC, 1994). No entanto, tal conduta, ocasiona mais traumas, frustrações e auto cobrança gerando estresse no indivíduo (SILVA et al., 2008).

O termo "estresse", com o significado usado na contemporaneidade, foi proposto pelo médico endocrinologista Selye, após ser demonstrado como uma resposta fisiológica ao enfrentar momentos de mudanças, frustrações, temores, situações ameaçadoras e angustiantes (SELYE et al., 1956). Com o constante estresse presente na vida de um indivíduo, as glândulas adrenais passam a produzir e liberar o cortisol, conhecido como o hormônio do estresse e sendo a causa de danos ao sistema imunológico, alterações de humor, elevação da pressão arterial e do risco de infarto (ZAVALIS et al., 2015; KOZASA, 2006; MANHN,2013). Deste modo, que a prática de Mindfulness, incentiva o meditador a aceitar seus pensamentos como algo natural, não os caracterizando como bons ou ruins, tornando a atenção do indivíduo ao momento presente e fazendo com que o mesmo sinta suas emoções e as sensações corporais que estão à sua volta (KABAT-ZINN, 1990).

É descrito na literatura, que a técnica tem a capacidade de moldar o cérebro, sendo possível a conquista da neuroplasticidade, trabalhando áreas como hipocampo e região têmporo-parietal, regiões responsáveis pela memória, aprendizado, empatia, compaixão e regulação das emoções (HOLZEL et al., 2011).

No Brasil, no ano de 2006 foi criado a Política Nacional de Práticas Integrativas e Complementares (PNPIC) (BRASIL, 2006), no qual foi possível implementar aos pacientes e comunidade que faz uso do Sistema Único de Saúde (SUS), cinco práticas, dentre elas: homeopatia e acupuntura. Após 10 anos da PNPIC, mais 14 práticas foram incorporadas à essa política, desta vez, utilizando também a técnica Mindfulness como ferramenta na terapêutica dos pacientes assistidos pelo Sistema Único de Saúde 
(BRASIL, 2017). De acordo com dados do Ministério da Saúde, no ano de 2017 foram registrados 1.4 milhão de atendimentos individuais em práticas integrativas e complementares, estima-se que somando as práticas coletivas, 5 milhões de pessoas sejam beneficiadas pelo programa por ano no SUS (BRASIL, 2018).

Ao longo do desenvolvimento regional, pode-se observar que a rotina de moradores de centros urbanos diverge de moradores de zonas rurais, desta forma foi determinado as localidades a serem analisadas, como é o caso de Taubaté e Monteiro Lobato (SOROKIN et al., 1981). O município de Taubaté durante o segundo império, destacou-se na produção de café no Vale do Paraíba, em seguida, sofreu graves crises econômicas que foram superadas e proporcionou com que a cidade se tornasse o centro industrial e possuir um forte setor comercial, além de ser uma cidade universitária e com uma importância significativa cultural (RICCI, 2004). A cidade de Taubaté, está localizada no eixo Rio-São Paulo, à $95 \mathrm{~km}$ do litoral norte e $45 \mathrm{~km}$ de Campos do Jordão, compondo uma integração entre o progresso e a natureza. Sendo estimada para o município, uma população de 311.854, proporcionando que a cidade estivesse entre as 100 cidades mais ricas do país e que concentram maiores bens e serviços produzidos, estando em $61^{\circ}$ lugar, a partir dos Censo Demográficos do IBGE (IBGE; 2018). Sendo uma das cidades mais desenvolvidas da região, a vida da população taubateana é proporcionalmente estressante. Em contrapartida, o município de Monteiro Lobato possui uma rotina prevalentemente mais calma, justamente por ser um município com menor população, cerca de 4.608 habitantes e com predomínio da população idosa, a cidade não possui trânsito e a população está concentrada na área rural, fatores que tornam a rotina menos estressante (ZERBINI et al., 2009). Com área de $332,74 \mathrm{~km}^{2} \mathrm{e}$ uma topografia montanhosa localizado na Serra da Mantiqueira e com domínios da Mata Atlântica em $32 \%$ de seu território, a cidade se desenvolveu com a predominância pecuária, notadamente leiteira favorecendo também o turismo rural (PIVOTT, 2014). A região localiza-se a $120 \mathrm{~km}$ da capital, com divisão com o Estado de Minas Gerais e limítrofe da cidade de Taubaté, a cidade teve a presença do escritor nomeia a cidade, Monteiro Lobato herdou a fazenda de seu avô e viveu no município de 1911 a 1917, no qual escreveu um de seus principais livros, Urupês, que reflete sua vivência na fazenda (LAZCANO, 2018).

O desenvolvimento e localização da cidade de Taubaté e Monteiro Lobato, caracterizam o padrão de vida dos habitantes, fatores externos que influenciam na qualidade de vida, uma vez que uma cidade mais desenvolvida, proporciona gatilhos de estresse. Devido ao potencial que a técnica possibilita aos praticantes, o objetivo desse estudo é o desenvolvimento de evidências iniciais na região para analisar quantitativamente o controle de estresse com a prática da técnica Mindfulness, entre duas cidades do Vale do Paraíba. 


\section{MATERIAL E MÉTODOS}

\subsection{Casuística}

Para a análise do controle de estresse e raiva com a prática da técnica Mindfulness foram utilizados um total de 20 participantes ( 8 homens e 12 mulheres), sendo 10 indivíduos do município de Taubaté e 10 indivíduos de Monteiro Lobato. Os indivíduos foram submetidos à realização de um questionário no início do projeto e ao final de 8 sessões semanais, com o tempo da prática Mindfulness com duração de 30 minutos, conduzido por um profissional biomédico especialista em medicina tradicional chinesa. As informações foram obtidas após terem sido especificados aos pacientes todos os procedimentos e estes terem assinado o termo de consentimento livre e esclarecido (TCLE). O projeto obteve aprovação do Comitê de Ética da Universidade do Vale do Paraíba (nº parecer 3197202/2019).

\subsection{Coleta de dados}

O processo inicial de abordagem aos indivíduos foi realizado presencialmente por meio de reuniões semanais. A aplicação dos questionários foi realizada por meio do projeto social autoral, desenvolvido pelo grupo de pesquisa, intitulado: "Medita Taubaté" e "Medita Monteiro" para avaliação de dados, realizado com moradores das respectivas cidades. Após assinado o termo de consentimento livre e esclarecido, os critérios de inclusão para participar desta pesquisa foram homens e mulheres, na faixa de 18 a 60 anos, de qualquer etnia, selecionados de forma aleatória por meio da disponibilidade de participação da pesquisa científica, totalizando 20 participantes, sendo 8 homens e 12 mulheres. Foram considerados critérios de exclusão: crianças, pessoas diagnosticadas com distúrbios do sistema endócrino, usuários de medicação para dormir, antidepressivos e corticóides. Também foram considerados não aptos para a avaliação: gestantes, mulheres que utilizam contraceptivos, indivíduos com processo inflamatório agudo, doentes mentais e deficientes intelectuais.

Para a realização da pesquisa, foram utilizados dois questionários distintos: obtenção de dados sócio demográficos desenvolvido pelos próprios pesquisadores para a caracterização amostral, e escala global de estresse perceptível (COHEN; KAMARCK; MERMELSTEIN, 1983). A escala de estresse é uma metodologia global, qualitativa ordinal, de auto resposta, contendo 6 perguntas que incluem a avaliação do grau de tristeza em reações inesperadas, grau de incapacidade de controle de vida, grau de sucesso, grau de confiança em resolução de problemas, controle de irritação e estresse e controle da própria vida. A avaliação se deu por respostas em score de 0 a 4 , que se deram por: ausência de estresse - score 0 , presença de estresse inicial - score 1 , presença levemente moderada de estresse - score 2, estresse moderado - score 3 , estresse intenso - score 4, onde foram analisados o grau de estresse do indivíduo antes e depois de serem submetidos à técnica de Atenção Plena (Mindfulness)durante o mês da realização desse estudo transversal. Para obtenção desses valores, o participante 
foi orientado a ser o mais sincero, visto que a percepção fosse computada de maneira fidedigna.

\subsection{Análise de dados}

A análise de dados foi realizado a partir do cálculo de scores obtidos do questionário (COHEN; KAMARCK; MERMELSTEIN, 1983), utilizou-se o software Excel® para organização e análise inicial, descritiva dos dados. As análises estatísticas foram realizadas no software GraphPad Prism 5.0 (GraphPad Software, San Diego, CA, USA, www.graphpad.com). Os resultados foram considerados, estatisticamente, significativos em $\mathrm{P} \leq 0,05$ por meio da análise do Teste de Pearson.

As características clínicas (idade, estado civil, presença de morbidades, quantidade de carga horária de trabalho semanal, presença de hobby, uso contínuo de medicamentos), foram computadas para descrição de dados sócio-demográficos da população estudada.

\section{RESULTADOS}

\subsection{Caracterização amostral}

A idade média da população analisada foi de 40 anos (25 - 59 anos, DP:24,04). De acordo com os dados coletados, $45 \%$ dos participantes possuem o estado civil de casado, $40 \%$ dos participantes são solteiros e $15 \%$ são divorciados. Dentre os participantes, $25 \%$ apresentaram alguma morbidade, $50 \%$ possuem como carga horaria mínima de trabalho 60 horas semanais, 35\% realizam no mínimo 40 horas semanais de trabalho e $15 \%$ dos pacientes realizam 20 horas semanais de trabalho. A presença de hobby praticada pelos participantes teve a prevalência de $50 \%$ e $45 \%$ dos participantes utilizam medicamentos de uso contínuo.

\subsection{Análise de dados}

No presente estudo, os resultados foram avaliados por score obtido pela metodologia de avaliação de estresse, antes e depois de serem submetidos à técnica de Atenção Plena (Mindfulness). A análise de estresse foi realizada utilizando um conjunto de seis perguntas onde obteve-se o score. A fim de caracterizar a prática da técnica Mindfulness em duas populações distintas, a análise estatística foi realizada em dois grupos, grupo 1: participantes da cidade de Taubaté e grupo 2: participantes da cidade de Monteiro Lobato.

Na análise 1, foi realizado a avaliação do grau de tristeza em reações inesperadas, com um grupo de dez participantes moradores da cidade de Taubaté (grupo 1), pré e pós submetidos à realização da prática de Atenção Plena, nesta análise, não foi 
observado diferença estatística significativa com a técnica, entretanto, é possível avaliar o potencial que a prática constante proporcionou nas reações emocionais $(P=0.066)$ (Figura 1.A). A análise 2, consistiu na avaliação do grau de incapacidade do controle de vida com um grupo de dez participantes moradores da cidade de Taubaté, pré e pós submetidos a realização da prática de Atenção Plena, nesta análise, foi observado diferença significativa da técnica Mindfulness no grupo após serem submetidos a prática constante $(P=0.005)$ (Figura 1.B). Na análise 3 , foi realizado a avaliação do grau de sucesso diante de momentos difíceis em um grupo de dez participantes moradores da cidade de Taubaté, pré e pós submetidos a realização da prática de Atenção Plena, nesta análise, não foi possível avaliar diferença estatística da técnica entre os grupos avaliados ( $P=0.431$ ) (Figura 1.C). A análise 4, baseou-se na avaliação da confiança em resolver os problemas pessoais, com um grupo de dez participantes moradores da cidade de Taubaté, pré e pós submetidos a realização da prática de Atenção Plena, nesta análise, não obteve diferença significativa da técnica Mindfulness ( $P=0.064)$ (Figura 1.D). Na quinta análise, foi realizado a avaliação do grau controle de irritação e estresse em um grupo de dez participantes moradores da cidade de Taubaté, pré e pós submetidos a realização da prática de Atenção Plena, observando diferença estatística significativa no grupo avaliado posteriormente à prática meditativa $(P=0.022)$ (Figura 1.E). Na análise 6 , realizado a avaliação do grau de controle da própria vida em um grupo de dez participantes moradores da cidade de Taubaté, pré e pós submetidos a realização da prática de Atenção Plena, observou-se o potencial da prática Mindfulness na modulação positiva das emoções ( $P=0.002)$ (Figura 1.F). 
Figura 1 - Correlação de scores obtidos em seis análises de participantes da cidade de Taubaté submetidos a técnica de Atenção Plena-Mindfulness (grupo 1). A: Análise 1. B: Análise 2, C: Análise 3, D: Análise 4, E: Análise 5, F: Análise 6.
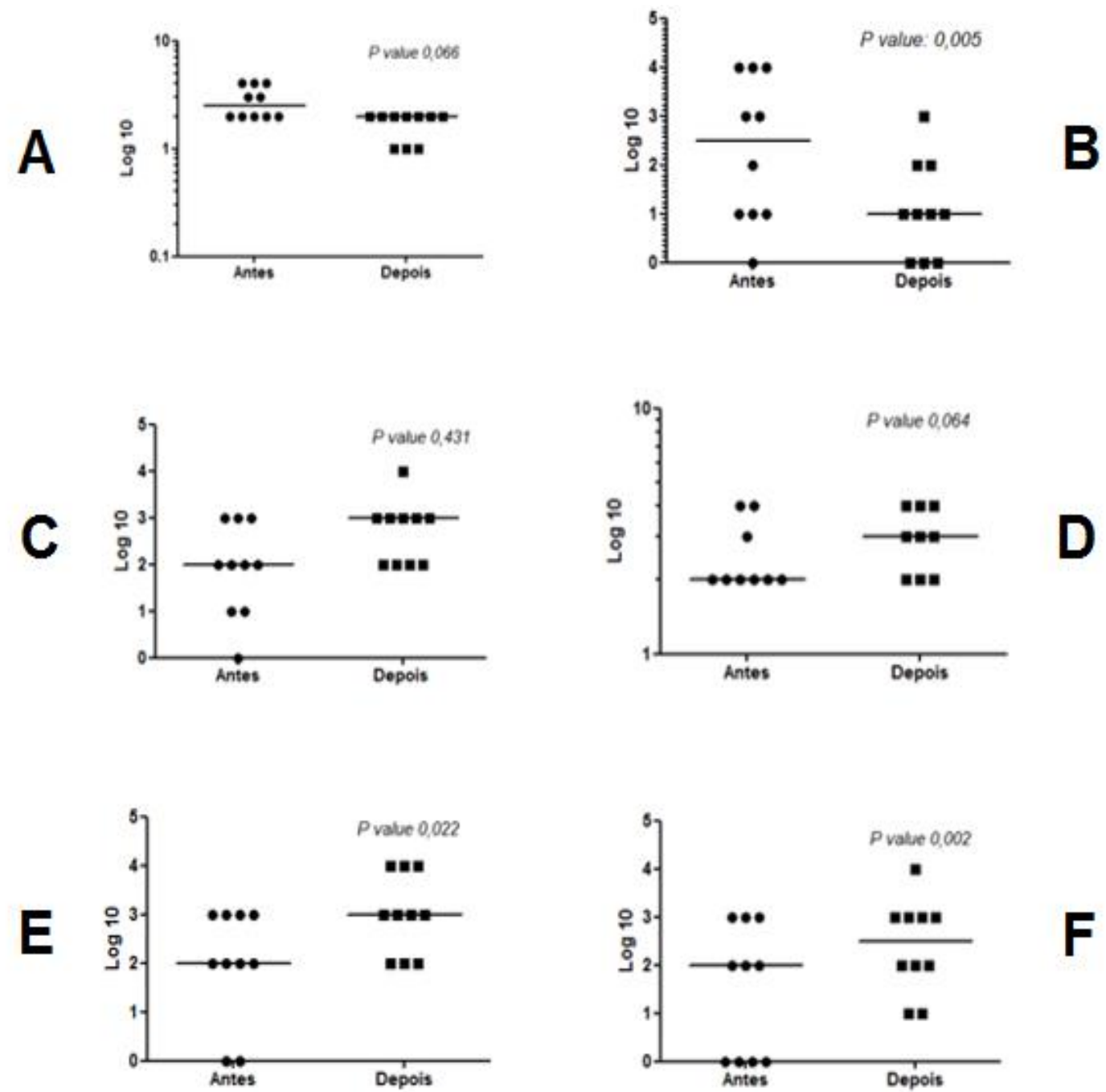

Fonte: As autoras.

Na cidade de Monteiro Lobato (grupo 2), foi realizada a análise em um grupo de dez pacientes pré e pós submetidos a técnica de Atenção Plena. Na análise 7 , avaliouse o grau de tristeza em reações inesperadas, não foi observado diferença estatística significativa com a técnica $(P=0.604)$ (Figura 2.A). A análise 8 , consistiu na avaliação do grau de incapacidade do controle de vida com um grupo de dez participantes moradores da cidade de Monteiro Lobato, pré e pós submetidos a realização da prática de Atenção Plena, nesta análise, não foi observado diferença significativa da técnica Mindfulness no grupo após serem submetidos a prática constante $(P=0.508)$ (Figura 2.B). Na análise 9 , foi realizado a avaliação do grau de sucesso diante de momentos difíceis em um grupo de dez participantes moradores da cidade de Monteiro Lobato, pré e pós submetidos a realização da prática de Atenção Plena, nesta análise, não foi possível avaliar diferença estatística da técnica entre os grupos avaliados ( $P=1.000)$ (Figura 2.C). 
A análise em que foi realizado a avaliação da confiança em resolver os problemas pessoais (Análise 10) com um grupo de dez participantes moradores da cidade de Monteiro Lobato, pré e pós submetidos a realização da prática de Atenção Plena, nesta análise, não obteve diferença significativa da técnica Mindfulness ( $P=0.153)$ (Figura 2.D). $\mathrm{Na}$ análise 11, foi realizado a avaliação do grau controle de irritação e estresse em um grupo de dez participantes moradores da cidade de Monteiro Lobato, pré e pós submetidos a realização da prática de Atenção Plena, observou-se a análise estatística significativa no grupo avaliado posteriormente a prática meditativa $(P=0.003)$ (Figura 2.E). A análise 12 , consistiu na avaliação do grau de controle da própria vida na população de Monteiro Lobato, pré e pós submetidos a realização da prática de Atenção Plena e não foi observado a diferença estatística significativa no grupo avaliado posteriormente a prática meditativa $(P=0.245)$ (Figura 2.F).

Figura 2 - Correlação de scores obtidos em seis análises de participantes da cidade de Monteiro Lobato submetidos a técnica de Atenção Plena-Mindfulness (grupo 2). A: Análise 7. B:

Análise 8, C: Análise 9, D: Análise 10, E: Análise 11, F: Análise 12.
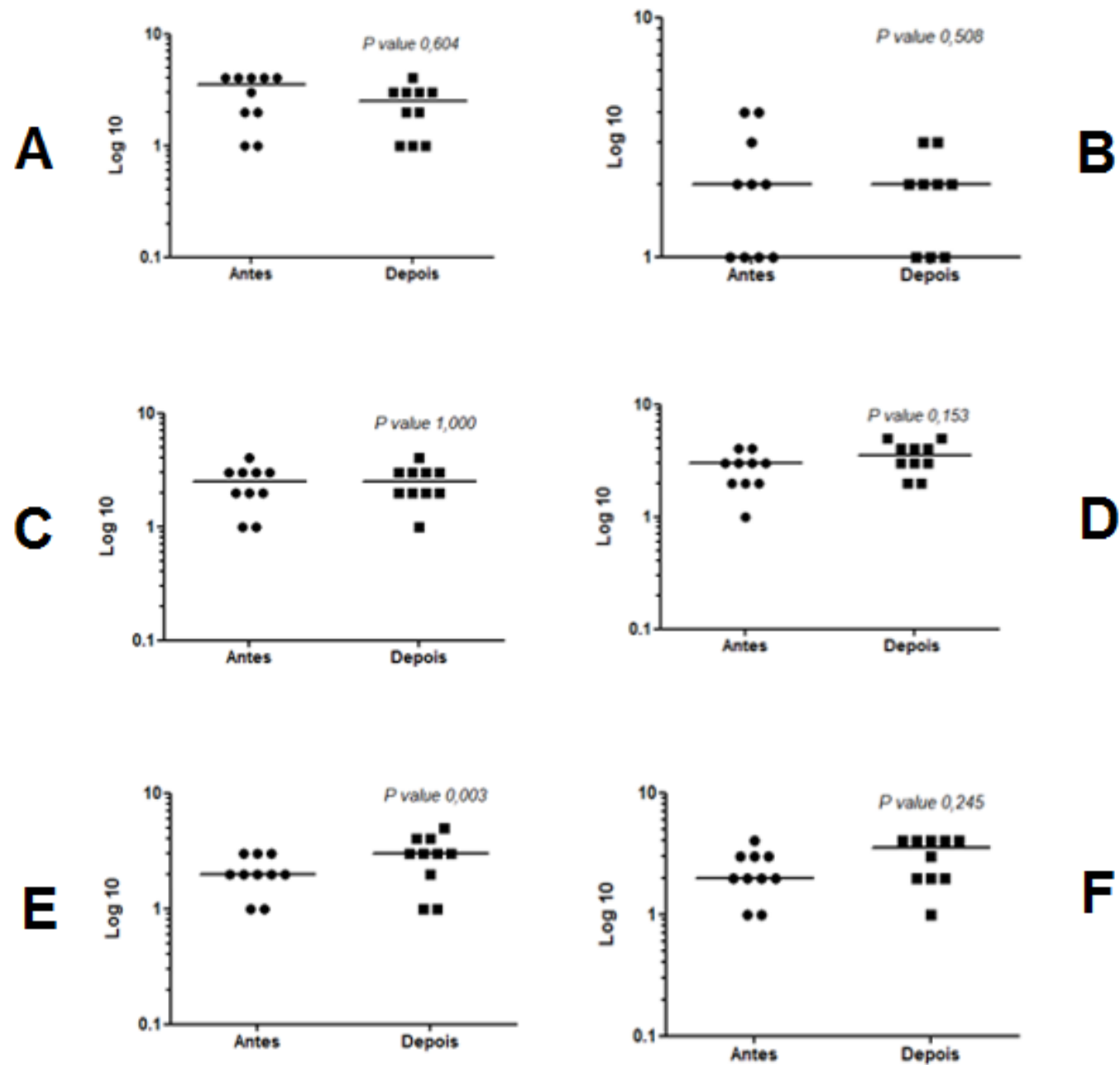

Fonte: As autoras. 


\section{DISCUSSÃO}

Dentre as análises de características observadas neste estudo, a avaliação do potencial de controle da vida na cidade de Taubaté, permitiu caracterizar que os indivíduos realizaram um melhor gerenciamento de suas próprias vidas, bem como o melhor domínio de seus sentimentos e atitudes perante momentos difíceis, enquanto no município de Monteiro Lobato, a evidência observada pelo atual estudo de caracterização de efeitos da prática Mindfulness, foi de um melhor controle da irritação e estresse. Baer et al. (2006) analisaram a prática contínua de Mindfulness pelo período de oito semanas, demonstrando que a prática apresenta baixas evidências em relação à redução de estresse, nível de atenção e humor. No entanto, a avaliação no presente estudo, quando avaliado a cidade de Monteiro Lobato, observa-se significativamente o controle da irritação e estresse $(P=0,003)$, fato que correlaciona com descritos na literatura utilizando o mesmo período de tempo, embasando o potencial da prática Mindfulness na região no Vale do Paraíba (GOYAL et al., 2014; GROSSMAN et al., 2004).

Embora não havendo estudos, se realizado a caracterização da prática Mindfulness com o potencial poder de modulação de estresse no período de oito semanas, na região do Vale do Paraíba, pode-se sugerir que a prática contínua da técnica Mindfulness poderá proporcionar uma redução nos efeitos maléficos que o estresse ocasiona na saúde dos indivíduos, entretanto, a confirmação requer estudos complementares (CARPENA; MENEZES, 2018). Os altos níveis desse hormônio, pode ocasionar consideráveis alterações no organismo como a resistência à insulina, produção de mediadores pró-inflamatórios e aumento da pressão arterial, gerando um desequilíbrio homeostático (HABIB et al., 2001; MARGIS et al., 2003; SOARES; ALVES, 2006).

Sabe-se que a impulsividade é descrita como um desejo de experimentação e capacidade de precipitação ao ato e a falta de atenção e o não planejamento, correlacionando com a capacidade de reflexão reduzida (MOREIRA, 2004; BARRATT, 1993). O autocontrole é associado a uma dificuldade do indivíduo o tornando incapaz de controlar suas vontades e adequá-las ao ambiente em que vive, bem como contornar e tomar as decisões mais funcionais diante das situações difíceis que surgem no cotidiano, a rotina acelerada em grandes cidades no qual o modo de rotina acelerado ocasiona o distanciamento do indivíduo de técnicas relaxantes (MCCRAE; COSTA JUNIOR, 1989; KABAT-ZINN, 1982; BAER, 2006).

Sendo assim, a prática da técnica Mindfulness tem como objetivo manter a atenção no momento presente, proporcionando a capacidade de realizar com o que o indivíduo faça reflexões e dê um passo atrás, analisando as situações que os confrontam para que melhores atitudes sejam tomadas, os resultados obtidos caracteriza um potencial que a técnica contínua quando aplicada nas duas populações 
analisadas, possui em auxiliar a redução de estresse e desta forma, proporcionando o autocontrole do indivíduo. Entretanto, a confirmação da hipótese requer estudos complementares, como a análise laboratorial de hormônios.

\section{CONCLUSÃO}

Devido às duas cidades do Vale do Paraíba avaliadas, possuírem características de desenvolvimento e cultura distintos, sendo o município de Taubaté com predominância da população na área urbana e Monteiro Lobato com predominância na área rural, os estilos de vida são diferentes. No entanto, por meio desse estudo de caracterização dos efeitos da prática Mindfulness em duas cidades do Vale do Paraíba, pode-se observar que a prática constante da técnica possui o potencial terapêutico, com benefícios ao praticante, contudo, para melhor compreensão bioquímica da ação no indivíduo, se faz necessário estudos complementares com abordagem complementares para determinação e quantificação dos benefícios na população brasileira. Os resultados desse estudo, demonstraram o potencial que a prática possibilitou nas duas amostras avaliadas, com a melhora nos indicadores de estresse após o período da prática $(P=0,022$ e $P=0,003)$, além disso, foi possível avaliar o potencial de controle que a prática proporcionou $(\mathrm{P}=0,002)$.

As análises dos dados revelam a necessidade da realização de análises em um maior número amostral, a fim de ser possível a validação dos resultados obtidos e avaliação das diferenças entre as variáveis que caracterizam a amostra. Este estudo caracteriza a técnica de Atenção Plena - Mindfulness, e sugere seu enorme potencial a ser utilizada na obtenção de uma melhor qualidade de vida e bem-estar.

\section{REFERÊNCIAS}

BAER, R. A. et al. Using self-report assessment methods to explore facets of mindfulness. Assessment, v. 13, n.1, p. 27-45, 2006

BAER, R. A. Mindfulness Training as a Clinical Intervention: A Conceptual and Empirical Review. Clinical Psychology: Science and practice.,v. 12, n. 2, p. 125-143, 2006.

BARRATT, E. S. Impulsivity: integrating cognitive, behavioral, biological and environmental data. In: MCCOWN, W. G.; JOHNSON, J. L.; SHURE, M. B. (eds.) The impulsive client: theory, research and treatment. [s.I]: American Psychological Association, 1993. p. 39-56.

BRASIL. Ministério da Saúde. Portaria n. 971, de 03 de maio de 2006. Aprova a Política Nacional de Práticas Integrativas e Complementares (PNPIC) no Sistema Único de Saúde. Diário Oficial, Brasília, 2006.

BRASIL. Ministério da Saúde. Portaria n. 849, de 27 de março de 2017. Inclui a 
Arteterapia, Ayurveda, Biodança, Dança Circular, Meditação, Musicoterapia, Naturopatia, Osteopatia, Quiropraxia, Reflexoterapia, Reiki, Shantala, Terapia Comunitária Integrativa e Yoga à Política Nacional de Práticas Integrativas e Complementares. Diário Oficial, Brasília, 2017.

BRASIL. Ministério da Saúde. Ministério da saúde inclui 10 novas práticas integrativas no SUS, 2018. Disponível em:

https://www.unasus.gov.br/noticia/ministerio-da-saude-inclui-10-novas-praticasintegrativas-no-sus. Acesso em: 26 Set. 2019.

CARPENA, M.A; MENEZES, C.B. Efeito da Meditação Focada no Estresse e Mindfulness Disposicional em Universitários. Psicologia: Teoria e Pesquisa. v. 34, 2018.

COHEN, S.; KAMARCK, T.; MERMELSTEIN, R. A global measure of perceived stress. Journal of Health and Social Behavior, v. 24, n.4, p. 385-396, 1983.

CRESWELL, J. D. Mindfulness interventions. Annual Review of Psychology, v. 68, p. $491-516,2017$.

GOYAL, Madhav et al. Meditation programs for psychological stress and wellbeing: a systematic review and meta-analysis. JAMA internal medicine, $v$. 174, n. 3, p. 357-368, 2014.

GROSSMAN, P. et al. Mindfulness-based stress reduction and health benefits: A meta-analysis. Journal of Psychosomatic Research. v. 57, n.1, p.35-43, 2004.

IBGE. Estimativas da população residente com data de referência 1 de julho de 2017: Taubaté. 2018. Disponível em: https://www.ibge.gov.br/cidadese-estados/sp/taubate.html. Acesso em: 10 abr. 2019.

KABAT-ZINN, J. Full catastrophe living: Using the wisdom of your body and mind to face stress, pain, and illness. New York: Delta, p.460, 1990.

KABAT-ZINN, J. An outpatient program in behavioral medicine for chronic pain patients based on the practice of mindfulness meditation: theoretical considerations and preliminary results. General hospital psychiatry, v. 4, n. 1, p. 33-47, 1982.

KOZASA, E. H. A prática de meditação aplicada ao contexto da saúde. Saúde Coletiva, v.3, n.10, p. 63-66, 2006.

HABIB, K. E. et al. Neuroendocrinology of stress. Endocrinology and Metabolism Clinics, v. 30, n. 3, p. 695-728, 2001.

HAYES, S. C. et al. What is acceptance and commitment therapy? In: HAYES S.C.; STROSAHL K.D. (eds). A practical guide to acceptance and commitment therapy. Boston: Springer, 2004. p. 3-29. 
HOLZEL, B. K. et al. How does Mindfulness Meditation Work? Proposing Mechanisms of Action from a Conceptual and Neural Perspective.

Perspectives on Psychological Science, v. 6, n. 6, p. 537-559, 2011.

LAZCANO, M. E. T. Tributos municipais e transferências constitucionais nos municípios de pequeno porte: estudo de caso Monteiro Lobato/SP. 2018. 48f. Trabalho de Conclusão de Curso (Especialização em Gestão Pública Municipal) - Universidade Tecnológica Federal do Paraná, Curitiba, 2018.

LINDSAY, E. K; CRESWALL, J. D. Mechanisms of mindfulness training: monitor and acceptance theory. Clinical Psychology Review, v. 51, p. 48-59, 2017.

LOPES, R. F. F.; CASTRO, F. S.; NEUFELD, C. B. A terapia cognitiva e o mindfulness: entrevista com Donna Sudak. Revista Brasileira de Terapia Cognitiva, v. 8, n. 1, p. 67-72, 2012.

MANHN, P. A. Ansiedade, qualidade do sono e concentração de cortisol salivar em tenistas adolescentes. 2013. 38f. Tese (Doutorado) - Universidade Estadual de Campinas, Piracicaba, 2013.

MARGIS, R. et al. Relação entre estressores, estresse e ansiedade. Revista de Psiquiatria do Rio Grande do Sul, v. 25, p. 65-74, 2003.

MCCRAE, R. R.; COSTA JUNIOR, P. T. More reasons to adopt the five-factor model. American Psychologist., v..44, n. 2, p. 451-452, 1989.

MOREIRA, D. F. Jogo patológico: análise por neuroimagem, neuropsicológica e de personalidade. 2004. 133f. Tese (Doutorado) - Universidade de São Paulo, São Paulo, 2004.

PIVOTT, C. A construção compartilhada de um plano de turismo para Monteiro Lobato, SP: a sustentabilidade como horizonte. 2014. 398f. Tese (Doutorado) - Universidade de São Paulo, São Paulo, 2014.

RICCI, F. O Desenvolvimento Regional e a Dinâmica do Movimento do Capital.

Revista Brasileira de Gestão e Desenvolvimento Regional. v. 1, n. 2, 2004.

ROEMER, L; BORKOVEC, T. D. Effects of suppressing thoughts about emotional material. Journal of Abnormal Psychology, v. 103, n. 3, p. 467-474, 1994.

SELYE, H. The stress of life. New York: McGraw-Hill, 1956.

SILVA, J. L. et al. Estresse e transtornos mentais comuns em trabalhadores de enfermagem. Revista Eletrônica de Enfermagem, v. 10, n. 4, 2008.

SOARES, A. J. A.; ALVES, M. G. P. Cortisol como variável em psicologia da saúde. Psicologia, Saúde \& Doenças, v. 7, n. 2, p. 165-177, 2006.

SOROKIN, et al. Diferenças fundamentais entre o mundo rural e o urbano. In: 
MARTINS, J. S. Introdução crítica à sociologia rural. São Paulo: Hucitec, 1981. p. 198-224.

ZAVALIS, Andrea et al. A influência dos fatores estressores sobre os níveis de atenção de profissionais de enfermagem. Revista de Pesquisa Cuidado é Fundamental Online, v. 7, n. 4, p. 3375-3387, 2015.

ZERBINI, T. et al. Trânsito como fator estressor para os trabalhadores. Saúde, Ética \& Justiça, v. 14, n. 2, p. 77-83, 2009. 